\title{
Dentate Gyrus and CA1 Ensemble Activity during Spatial Reference Frame Shifts in the Presence and Absence of Visual Input
}

\author{
Katalin M. Gothard, ${ }^{1}$ Kari L. Hoffman, ${ }^{2}$ Francesco P. Battaglia, ${ }^{2}$ and Bruce L. McNaughton ${ }^{2}$ \\ ${ }^{1}$ Department of Psychiatry, California Regional Primate Research Center, University of California Davis, Davis, California \\ 95616, and 2Arizona Research Laboratory Division of Neural Systems, Memory and Aging, University of Arizona, Tucson, \\ Arizona 85724
}

In rats shuttling between a variably placed landmark of origin and a fixed goal, place fields of hippocampal CA1 cells encode location in two spatial reference frames. On the initial part of the outbound journey, place fields encode location with respect to the origin while on the final segment, place fields are aligned with the goal (Gothard et al., 1996b). An abrupt switch of reference frame can be induced experimentally by shortening the distance between the origin and the goal. Two linked hypotheses concerning this effect were addressed: (1) that the persistent, landmark-referenced firing results from some internal dynamic process (e.g., path integration or "momentum") and is not a result of maintained sensory input from the landmark of origin; and (2) that this hypothetical process is generated by connections either within CA3 or between CA3 and
CA1, in which case the effect might be absent from the dentate gyrus. Neuronal ensemble recordings were made simultaneously from CA1 and the dentate gyrus as rats shuttled on a linear track between a variably located box and a goal, under light or dark conditions. The box-referenced firing persisted significantly longer in the dark in both hippocampal subfields, suggesting a competitive interaction between an internal dynamic process and external sensory cues. The similarity between reference frame transitions in the dentate gyrus and the CA1 region suggests that this process probably occurs before CA3, possibly in the entorhinal cortex or subiculum.

Key words: multisite recording; path integration; spatial memory; navigation; neural ensembles; population vector; granule cells
Both environmental and self-motion cues contribute to placespecific firing in the rat hippocampus. In certain tasks, visual cues control the location of CA1 place fields (O'Keefe and Conway, 1978; Hill and Best, 1981; Muller and Kubie, 1987; Knierim et al., 1995; O'Keefe and Burgess, 1996). Nevertheless, place fields can be generated and maintained in darkness (O'Keefe, 1976; McNaughton et al., 1989; Quirk et al., 1990; Markus et al., 1994). In darkness, self-motion cues (vestibular and motor efference signals) are sufficient to update the angular component of hippocampal location information via a process called path integration (Mittelstaedt and Mittelstaedt, 1982; Etienne et al., 1996; Golob and Taube, 1999). The spatial information conveyed by visual and self-motion cues is normally redundant; however, an experimentally induced conflict between these two types of cues can disambiguate their relative contribution to place cell activity (Gothard et al., 1996a,b; Bures et al., 1997; Knierim et al., 1998). Rats trained to shuttle on a linear track between a start box and a fixed goal at the opposite end of the track experience an inconsistency between their location relative to the fixed cues and the shifted box. Under these conditions, at least on the initial segment of the journey, place cells fire with an invariant spatial relationship to

\footnotetext{
Received Aug. 24, 2000; revised June 15, 2001; accepted July 5, 2001.

This work was supported by National Institutes of Health Grant NS20331 (K.M.G. and B.L.M.), by Human Frontier Science Program Grant LT0150/1999-B (F.P.B.), and by a National Science Foundation predoctoral fellowship (K.L.H.). We thank Shanda Roberts and Karen Weaver-Sommers for help with recording and James. J. Knierim and A. David Redish for helpful comments on this manuscript.

Correspondence should be addressed to Dr. Bruce L. McNaughton, 384 Life Sciences North, University of Arizona, Tucson, AZ 85724. E-mail: Bruce@ nsma.arizona.edu.

Copyright (C) 2001 Society for Neuroscience $\quad 0270-6474 / 01 / 217284-09 \$ 15.00 / 0$
}

the box (Gothard et al., 1996b). This strongly suggests that, on the initial segment of a journey, the surrounding visual cues do not provide the major driving force for place cell firing; rather, location is updated by either self-motion signals or intrinsic dynamics of the hippocampal and related circuitry. A study by Gothard et al. (1996b), however, left open the possibility that the maintained alignment of the place fields with respect to the start box was a result of the continued availability of visual information about the rat's position relative to the box. A primary goal of this study was, therefore, to determine whether place fields remain aligned with the box in the dark and whether the dynamic interaction between linear self-motion signals and external sensory cues in the dark is biased in favor of self-motion signals.

Little is known about how and where in the brain the path integration operation takes place. Lesion studies (Alyan and McNaughton, 1999; Maaswinkel et al., 1999) have led to conflicting conclusions as to whether the hippocampus performs this computation or whether it occurs elsewhere and the hippocampus merely receives the result (Cooper and Mizumori, 1999; Redish, 1999). One possibility is that the effect occurs directly within CA3 recurrent collaterals (Samsonovich and McNaughton, 1997), in which case one might predict differences in the behavior of CA1 pyramidal cells and dentate gyrus granule cells. The secondary goal of this study was, therefore, to compare place field shifts in the dentate gyrus and CA1 induced by systematically shifting the point of departure of the journey. If the updating of the representation based on self-motion signals involves a computation that takes place primarily within the CA3 region, and the result is not fed back to the hippocampal input (i.e., open loop processing), then one might expect differences in place field shifts between the dentate gyrus and CA1. 


\section{MATERIALS AND METHODS}

Surgery, electrode assembly, and recording technology. Surgery was conducted according to National Institutes of Health guidelines for rodents. Seven adult male Fisher 344 rats were implanted under pentobarbital anesthesia with a circular array of 14 separately moveable microdrives. This device and the parallel recording technique were described first by Wilson and McNaughton (1993) and in more detail by Gothard et al. (1996a). Briefly, each microdrive consisted of a drive screw coupled by a nut to a guide cannula. Each guide cannula contained a tetrode, a four-channel electrode constructed by twisting together four strands of insulated $13 \mu \mathrm{m}$ nichrome wire (H. P. Reid, Inc., Neptune, NJ). A full turn of the screw advanced the tetrode $318 \mu \mathrm{m}$. The stereotaxic coordinates for the placement of the electrode array were 1.8 or $2.0 \mathrm{~mm}$ lateral and $3.8 \mathrm{~mm}$ posterior to bregma on the right hemisphere. The tetrodes were lowered gradually after surgery into the hippocampus and allowed to stabilize above the CA1 layer or the upper blade of the dentate gyrus. Two of the tetrodes served as reference electrodes. The four channels of each tetrode were attached to a 50-channel unity-gain head stage (Neuralynx Inc., Tucson AZ). The rat's position was recorded by tracking two clusters of infrared diodes. A large cluster of diodes was mounted on the head stage, and a smaller cluster of diodes was mounted on a $14 \mathrm{~cm}$ lightweight aluminum rod attached to the back of the head stage to monitor head orientation. The position and head orientation of the animal were recorded with a sampling frequency of $20 \mathrm{~Hz}$.

A multiwire cable connected the head stage to a commutator (Biela Idea Development, Anaheim, CA) from which the signals were transmitted to digitally programmable amplifiers (Neuralynx). The signals were amplified by a factor of 10,000, bandpass-filtered between $600 \mathrm{~Hz}$ and 6 $\mathrm{kHz}$, and transmitted to an array of seven 486 computers equipped with synchronized clocks. Signals crossing a manually set threshold were digitized at $32 \mathrm{kHz}$. Spikes were sorted off-line on the basis of the relative amplitude and other characteristics of action potentials (e.g., spike width and area under the curve) on different tetrode channels (McNaughton et al., 1983; Recce and O'Keefe, 1989) using custom software (Wilson and McNaughton, 1993).

Behavioral apparatus and task. The behavioral apparatus was a $184 \times$ $8 \mathrm{~cm}$ wooden linear track, painted black. The track was placed in the center of a large room and surrounded by black curtains. Large white objects hung in front of the curtains and asymmetrically aligned lights served as additional static background cues. A small food well was mounted at one end of the track. Attached to the other end was a 27-cm-high, 32-cm-wide, 27-cm-long box, which could be repositioned along the track. The box had carpeted floors and a centrally located food well. Rats were trained to shuttle along the full length of the track between the two food wells. To vary the journey length, the box was moved to one of five equally spaced locations along the track (Fig. $1 \mathrm{~A}$ ). These five locations are referred to as box 1-box 5, with box 1 out being the longest box outbound journey (box located at the far end of the track). The distance from the front edge of the box to the fixed food well varied from $151 \mathrm{~cm}$ for the longest journey to $53 \mathrm{~cm}$ for the shortest journey (in the box 1 position, the back of the box extended beyond the edge of the track by $5 \mathrm{~cm}$ ).

All rats were initially trained with the box in position 1 . The first trial of each recording session always started from the box 1 position. While the rat was traveling from the box to the goal, the box was moved to one of the five possible locations along the track using a pseudorandomized blocks schedule (i.e., on each block of five trials, positions 1-5 were sampled without replacement). The rat ate the food reward placed in the fixed food well and returned to the box, which by then had been moved to a new location. Thus, each trial started where the previous trial had ended, and the position of the box was typically different at the beginning and the end of each trial. A recording session consisted of 10-15 blocks of five positions. Event flags, inserted automatically into the data file, marked the time of each box exit and box entry and also the time of reaching the fixed food well and departing from it. These event flags permitted the selection and separate analysis of all five types of outbound and inbound journeys without including the segments while the rat was eating at either food well. The apparatus and the randomization method were described in detail by Gothard et al. (1996b).

Training. The food reward was a mixture of mashed rat chow, applesauce, and a veterinary nutritional supplement (Stat; PRN Pharmacal, Inc.). On the initial 3-5 d of habituation to the room and the track, the rats were placed inside the box, always in the box 1 position, and allowed to explore. Rats ate drops of food strewn along the track as they made excursions of increasing length from the box. Eventually, they traveled
A
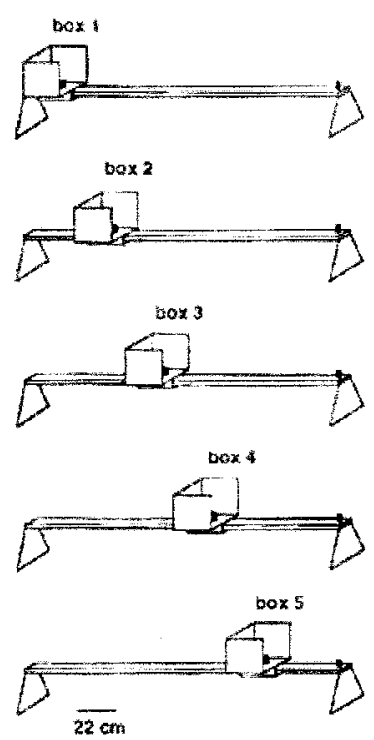

$c$

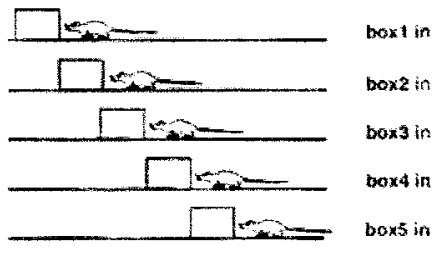

Figure 1. Behavioral apparatus and journey types. A, A $27-\mathrm{cm}$-high, $32 \times$ $27 \mathrm{~cm}$ moveable cardboard box was mounted on a $184 \times 8 \mathrm{~cm}$ linear track. The box was moved to one of five possible locations along the tack (box 1-box 5). A small food cup was mounted in the box, and another cup was permanently mounted at the opposite end of the track (goal). The rat was trained to exit the box and run to the goal (outbound journey). As the rat approached the goal, the box was shifted to a new location. After consuming the reward at the goal, the rat returned to the box (inbound journey). During the subsequent outbound journey, the box was shifted to a new location. $B$, The five types of outbound journeys (box1 out-box5 out). $C$, The five types of inbound journeys (box1 in-box 5 in).

the whole length of the track multiple times in a session. Each rat was trained to run at least 40 consecutive trials. Four of the seven rats were trained under these conditions, which henceforth are referred to as the light condition. Three other rats were also trained to a criterion of 40 trials with the lights turned off (henceforth the dark condition). The recording room had black floors, walls and ceiling, and double doors, which prevented light from entering. Behavioral monitoring by the experimenter was accomplished using a deep infrared light source and night vision goggles. When the rats would run $>40$ consecutive trials in either condition, they were implanted with the multielectrode device described above.

After recovery from surgery, the rats received additional training, and the tetrodes were gradually lowered into the CA1 pyramidal layer or the granular layer of the dentate gyrus. During the entire training period and during the first one or two recording sessions, the box was always maintained in its initial position (box 1). At this stage, the start box, the linear track, the fixed food cup, and the surrounding objects constituted a single, fixed reference frame. When the box was moved, it defined a new reference frame, whose alignment with the laboratory frame was variable.

Cell classification. During the process of optimizing tetrode placement, the EEG and spontaneous unit activity were monitored on each tetrode. The buildup of clusters of spikes was monitored as accumulating points in a scatter plot. Each point on the scatter plot corresponded to the height of the same spike registered on two of the four channels of the tetrode. Principal cells in the CA1 region were distinguished from interneurons on the basis of spike width $(>250 \mu$ sec measured from the negative to the positive peak; mean, $350 \mu \mathrm{sec})$, the property of firing complex spikes, and an overall mean rate of $<5 \mathrm{~Hz}$ (Ranck, 1973). CA1 pyramidal cells were recorded from tetrodes that registered sharp waves and "ripples" (Buzsaki, 1986) during sleep and quiet wakefulness.

All tetrodes in the dentate gyrus (DG) registered $\gamma$ oscillations $(30-60$ Hz; Stumpf, 1965; Buzsaki et al., 1983; Bragin et al., 1995). When the electrodes reached the layer at which clear $\gamma$ oscillations were observed, they were advanced in small increments until single-unit activity was observed. The recorded cells were divided, on the basis of firing rates, 


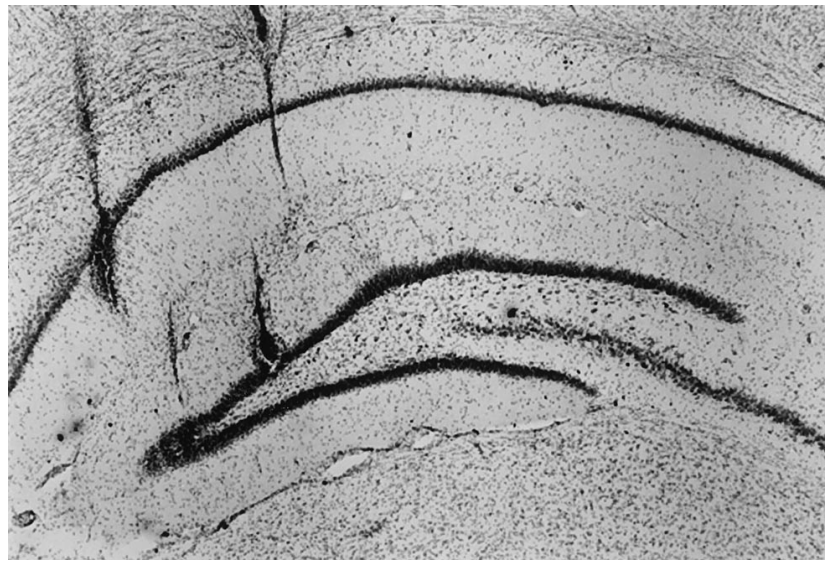

Figure 2. Histological verification of electrode placement. A coronal section of the hippocampus stained with cresyl violet is shown. For each rat, one tetrode, assumed on physiological grounds to be in the CA1 pyramidal layer, and a second tetrode, assumed to be in the granular layer of the DG (see Materials and Methods), were selected. Lesions were produced after the final recording session by passing $5 \mu \mathrm{A}$ of cathodal current through a tetrode for $5 \mathrm{sec}$. Additional electrode tracks are visible above the two lesions, medial to the dentate gyrus lesion and lateral to the CA1 lesion. In this rat, all the electrode tracks were medial to the CA3c region, thus ruling out misidentification of CA3 pyramidal cells as PG cells.

into two groups. Cells with firing rates of $>2.5 \mathrm{~Hz}$ were classified as high-rate interneurons (Jung and McNaughton, 1993; Freund and Buzsaki, 1996; Nitz and McNaughton, 1999). Cells with firing rates of $<2.5 \mathrm{~Hz}$ were grouped as a single category. This group may have included not only granule cells but also some mossy cells and low-rate interneurons. No additional discrimination between these cells types was possible on the basis of extracellular recordings; however, granule cells outnumber the other cell classes in the dentate gyrus by a factor of $\sim 10$. Therefore the term putative granule (PG) cell is used throughout the remainder of this report with the explicit caveat that the PG group may not have consisted entirely of granule cells.

High-rate interneurons in the dentate gyrus formed tightly packed, dense clusters in the spike amplitude scatter plots. Usually more than one interneuron was recorded on the same tetrode. PG cells discharged single spikes or small bursts, typically had low average rates $(<1 \mathrm{~Hz})$, and generated less compact parameter clusters than high-rate interneurons.

At the end of the experiment, a small electrolytic lesion was made at the tips of two of the tetrodes. One of these tetrode tips was at a depth estimated to be in CA1, registering EEG and single-unit signals characteristic of the CA1 region. The second tetrode was at a depth estimated to be in the dentate gyrus, having passed through the estimated CA1 pyramidal layer and just beyond the region of maximal $\theta$ amplitude. In one rat, a lesion was placed in a region estimated to be the CA3 pyramidal cell layer between the upper and lower blades of the dentate gyrus. The lesions were visualized histologically in cresyl violet-stained $40-\mu \mathrm{m}$-thick slices. Each lesion was localized to the intended area, confirming that the electrophysiological criteria used to estimate the recording sites were reliable. In one rat, all tetrodes were medial to the most lateral aspect of the CA3 region (Fig. 2). The low-rate cells from this rat, which were recorded from depths corresponding to the upper and lower blades of the dentate gyrus, and all of the cells recorded in the other rats at sites of dentate gyrus lesions were selected for the illustrative examples of PG cells. There is no question that these cells do not include CA3c pyramidal cells. The properties of these cells were indistinguishable from the remainder of the PG class.

Data analysis. The distribution of population activity along the track was calculated using a sparsity index (based on cells that fired with a mean rate of $>0.35 \mathrm{~Hz}$ during track running). For each of the 64 bins into which the track was subdivided, the sparsity is defined as:

$$
a=\left(1 / N_{\mathrm{c}}\right)\left(\Sigma_{\mathrm{I}} f_{\mathrm{I}}\right)^{2} /\left(\Sigma_{\mathrm{I}}^{2} \mathrm{I}_{\mathrm{I}}^{2}\right)
$$

where $f_{\mathrm{I}}$ is the average firing rate for the $I$ th cell in that bin, and $N_{\mathrm{c}}$ is the number of cells. For a completely distributed representation, i.e., one in which all the cells fire at the same rate in one location, the sparsity has a value of 1. For the sparsest possible representation, i.e., a representation in which only one cell fires and the others are silent, the sparsity takes the minimum allowed value of $1 / N_{\mathrm{c}}$. Note, however, that because inactive cells were not included, this measure is strictly a means of determining whether there were spatial variations in population activity and cannot be used to compare the general sparsity of the ensemble representations in the CA1 and DG regions.

To determine whether place cells fire at fixed distances from the variably placed box, the place field shift was estimated by calculating the center of mass of the locations at which spikes occurred for each neuron and each journey type. The center of mass is computed as the average of all the locations on the rail at which a spike occurred and provides an estimate of place field location that is independent of firing rate and place field size or shape. If a cell fired fewer than three spikes on a journey type, the center of mass was not calculated for that journey type. If a cell had multiple place fields, the center of mass will be at the average location of the subfields. For cells recorded in multiple sessions, only one session was selected for these calculations. The centers of mass were plotted on a scaled drawing of the track for all five types of outbound journeys (see Fig. 5). For each neuron, the centers of mass across journey types were connected by lines, providing an indication of the degree to which place cells were aligned with the box or the fixed cues as a function of distance along the track.

Construction of population vectors and vector correlation matrices. The $176 \mathrm{~cm}$ distance between the food well in box 1 and the fixed food well at the opposite end of the rail (the full extent of the journey traveled by the rat) was divided into $642.75 \mathrm{~cm}$ spatial bins. The firing rate of each cell was computed for each bin, for each outbound journey type (box 1, box 2 . . box 5), for each condition (light vs dark), and for each region (CA1 vs DG). Data from all available recording sessions were combined for this analysis, but if the same cell appeared to have been recorded in multiple sessions, data from only one of these sessions were used. The $N$-dimensional vector (where $N$ is the total number of included cells recorded in dark or light in CA1 or DG) represents the population firing pattern at a specific location averaged across rats (Fig. 3).

A population vector for a given spatial bin represents the list of firing rates for all the recorded cells on that journey type, condition, and hippocampal subregion. For example, the PG population vector for the 10th spatial bin $(27 \mathrm{~cm}$ from the exit of box 1) on the box 1 outbound journey in the light condition contained a list of firing rates for that location from all 125 recorded PG cells that showed outbound activity anywhere on the track. The population vector for the same spatial bin for the box 2 outbound journeys contained the firing rates of the same 125 PG cells when the rat was exiting the box in the box 2 position. The difference between the two conditions under which these population vectors were recorded is that even though the rat was in the same spatial bin, during the box 2 outbound journey the rat was just exiting the box at that location, whereas in the box 1 outbound journey, the rat was $27 \mathrm{~cm}$ from the box. A different population vector was compiled for the same spatial bin from 79 PG cells recorded in the dark. Vector correlations were used to compare the population-firing pattern at different points on the track, across different outbound journey types. The correlation coefficient, that is, the normalized overlap, of each pair of population vectors was calculated and used as a measure of the similarity of population firing patterns at different locations on different types of journeys. The correlation coefficient was defined as:

$$
\tau_{\mathrm{ab}}=\frac{\sum_{\mathrm{i}} S_{\mathrm{i}}(a) S_{\mathrm{i}}(b)}{\sum_{j} S_{\mathrm{j}}^{2}(a) \sum_{\mathrm{k}} S_{\mathrm{k}}^{2}(b)},
$$

where $S_{\mathrm{i}}(a)$ is the firing of the $i$ th cell at location $a$.

Coherency and reference frame transition analysis. For the population vector analysis, which provides a means of visualizing the reference frame switch along the track (see Fig. 6), data were averaged across multiple trials, recording sessions, and rats. This analysis does not provide information about the population dynamics on a single-trial basis, making it difficult to assess the statistical significance of any effects that may be observed. Redish et al. (2000) presented a method to compute the extent to which the internal representation contained in the firing of hippocampal cells is tied to different reference frames at any given moment in time, in an experimental context similar to the one used here. With this method, it is possible to estimate the point at which the representation transits from the box-related frame to the room frame. The details of this method have been described by Redish et al. (2000). 

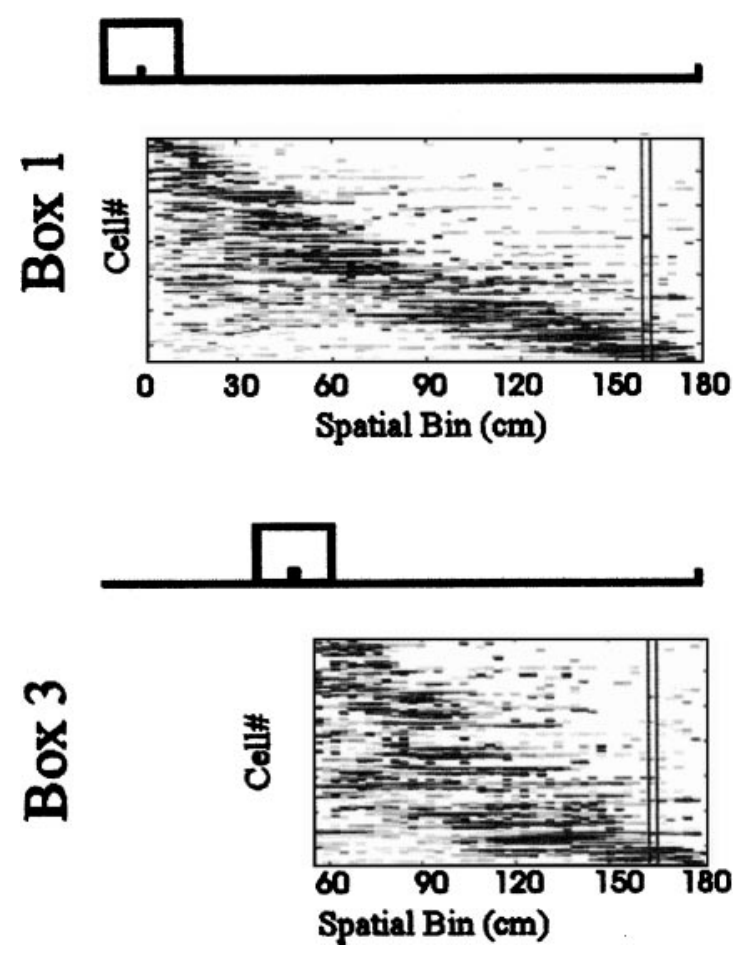

Figure 3. Construction of population vectors. Population activity of an ensemble of 130 cells from CA1 in the light condition along the full-length journey (top panel) and a shortened journey (bottom panel) is shown. The $x$-axis represents the 64 spatial bins in which firing rates were computed for each cell, marked as distance (in centimeters) along the rail. Each row on the $y$-axis represents the firing rate of a cell in each $2.75 \mathrm{~cm}$ spatial bin. The firing rate scale goes from white (no firing) to black (high firing). Cells are sorted according to the firing profile center of mass calculation. A population vector was defined as the list of the firing rates for the corresponding spatial bin. For illustration, one such bin is highlighted for each journey type. The spatial bin size was kept constant across journey types.

Briefly, a reconstruction procedure is used to determine the internal representation of the rat's position contained at any point in time in the ensemble activity. Given the results of the averaged population vector analysis, at the beginning of an outbound journey, it could be expected that the reconstruction of the position in the box frame of reference would be more accurate than the reconstruction of the position in the room frame. As the reference frame transition occurs, the quality of the reconstruction in the box frame will decrease, and the quality of the reconstruction in the room frame will increase until it becomes better than the quality of the reconstruction in the box frame. The transition point was defined as the location at which this occurred. If visual information from the fixed reference frame contributes to the transition, then the transition should occur at a shorter distance from the origin in the light condition compared with the dark condition. To test this hypothesis, the distributions of transition points across trials in light and dark conditions were computed and compared. To reconstruct the rat's position, the activity packet defined as:

$$
A_{\mathrm{t}}^{\mathrm{C}}(v)=\frac{\sum_{\mathrm{i}}\left(F_{\mathrm{i}}(t) \cdot S_{\mathrm{i}}^{\mathrm{C}}(v)\right)}{\sum_{\mathrm{i}} F_{\mathrm{i}}(t)},
$$

was computed (Samsonovich and McNaughton, 1997), where $F_{\mathrm{i}}(t)$ is the firing of the $i$ th cell at time $t, S_{\mathrm{i}}{ }^{\mathrm{C}}(v)$ is the number of spikes fired, over all trials, by cell $\mathrm{i}$, when the animal was in position $v$ in the reference frame C. Thus, if all the cells had very small and reliable place fields in one frame, the activity packet would be confined to a narrow spatial region; however if place fields were widespread and unreliable, the activity packet would be wider. The coherency of the activity packet, defined as:

$$
K^{\mathrm{C}}(t)=\sum_{\mathrm{v}}\left(A_{\mathrm{t}}^{\mathrm{C}}(v) \cdot G_{\mathrm{u}}(v)\right)
$$

where $G_{\mathrm{u}}(v)$ is an idealized gaussian activity packet centered in the rat's actual position, with a fixed width $(9 \mathrm{~cm})$, is a measure of the accuracy of the reconstruction in the frame $\mathrm{C}$.

For each trial, the coherencies in the two frames were computed at all the locations sampled by the rat. Each trial was subdivided into 20 time bins, and the coherency of the activity packet was computed for each time bin if at least one spike occurred in that time bin. To determine the transition point, the difference of the coherencies in the room frame and in the box frame were computed. The point at which the difference crossed zero is defined as the transition point. When there was more than one transition point, the zero crossing corresponding to the absolute minimum of the function was taken.

For the transition point analysis, data were used from 11 sessions from three rats in the dark condition and 17 sessions from five rats in the light condition. For the light condition, coherency values were computed for 1911 trials ( 423 box 1 trials, 383 box 2 trials, 374 box 3 trials, 372 box 4 trials, and 359 box 5 trials). For the dark condition, coherency values were computed for 518 trials (109 box 1 trials, 101 box 2 trails, 103 box 3 trials, 103 box 4 trails, and 102 box 5 trials). The transition points were computed only for those trials for which the coherency was defined (see Materials and Methods, coherency and reference frame transition analysis) in at least 15 of 20 time bins.

A total of 892 transition points were calculated in the light condition (231 transition points for box 1 trials, 188 for box 2 trials, 156 for box 3 trials, 177 for box 4 trials, and 140 for box 5 trials). A total of 180 transition points were computed in the dark condition (52 transition points for the box 1 trials, 43 for the box 2 trials, 34 for the box 3 trials, 30 for the box 4 trials, and 21 for the box 5 trials).

\section{RESULTS}

All 7 rats acquired the task in $\sim 10-15$ training sessions in the light. An additional 15-20 sessions were required to acquire the task in the dark. Rats ran slower in the dark (mean \pm SD velocity, $29.07 \pm 8.42 \mathrm{~cm} / \mathrm{sec}$ ) than in the light (mean velocity, $37.81 \pm 10.7$ $\mathrm{cm} / \mathrm{sec}$; two-tailed $t$ test, $p<0.0001)$. In the dark, the lower running velocity was not accounted for by excessive hesitation in the vicinity of the goal. The rats did not show a tendency to overshoot the goal or to fall off the track. To test whether the olfactory or tactile cues associated with the baited food cup could account for the precise localization of the goal in the dark, a probe trial was administered, in which a baited food cup, identical to the one at the goal, was placed in the middle of the track, 30-50 $\mathrm{cm}$ closer to the box than the true goal. The rat ran over, passed the food cup in this novel location, and stopped at the expected goal location.

When the box was moved for the first time in either condition, the rats hesitated before entering the box and explored vigorously. In the next two or three trials, however, they habituated to the manipulation and showed no additional hesitation to enter the box. Data from sessions when the box was moved for the first time were excluded from analysis. The duration of a trial was $\sim 25 \mathrm{sec}$, including the time that the rat spent eating in the box and at the fixed food well. In a recording session of 50-75 trials, each start box location was sampled $10-15$ times. The session came to an end when the rats slowed down. The number of recording sessions per rat varied between 6 and 25; more recording sessions were possible when each electrode yielded a good-quality signal in both the CA1 and the dentate region, and fewer sessions were possible when the electrodes that were aimed at the dentate gyrus did not encounter recordable signals.

A total of 1821 spike trains, from units considered well isolated, were recorded from seven rats. From these, 240 spike trains, with average rates of $<0.03 \mathrm{~Hz}$, were eliminated from analysis. Because of similarities in spike waveform, relative spike height ratio on the four tetrode channels, and spatial selectivity across sessions, some cells were assumed to have been recorded from over 
two or more sessions. For each of these cells, the session showing the best signal-to-noise ratio (largest spikes) and cleanest unit isolation was used for the population vector analysis. After excluding high-rate interneurons (278 spike trains, of which 187 were recorded from CA1 and 91 were recorded from the dentate gyrus) and cells recorded multiple times (798 spike trains), a total of 505 cells were analyzed. Of these, 301 cells were recorded from the CA1 region, 144 of which were recorded in the light condition and 157 of which were recorded in the dark condition. Of the 204 PG cells, 125 were recorded in the light condition, and 79 were recorded in the dark condition. The average firing rates of the CA1 pyramidal cells in the light and dark conditions were $0.97 \pm$ 0.74 and $0.95 \pm 0.68 \mathrm{~Hz}$, respectively. The average firing rates for PG cells in the light and dark conditions were $0.73 \pm 0.55$ and $0.74 \pm 0.58 \mathrm{~Hz}$, respectively. For the trial-by-trial analysis of transition points on the outbound journey, all spike trains with more than three spikes on the outbound journey were used.

No difference in place field distribution along the track was observed between cells recorded from the CA1 region and the dentate gyrus, and no spatial trends in the sparseness of the distributions were observed, meaning that the place fields were approximately uniformly distributed. Finally, no significant differences in spike width between CA1 and PG cells were found (CA1 spike width, $358 \pm 47 \mu$ sec; DG spike width, $328 \pm 66$ $\mu \mathrm{sec})$.

\section{Comparison of place field shift in CA1 and DG}

CA1 pyramidal cells and putative granule cells responded similarly to the variations in the start box location. In both CA1 and DG, place fields located immediately after exiting the box remained at fixed distances from the box (Fig. 4) in both light and dark conditions. Place fields near the opposite end of the track did not appear to be influenced by the position of the box, remaining fixed in the reference frame of the track and the surrounding static cues. Place fields between these two regions exhibited transitional properties. In this transition zone, especially in the most shortened journeys, some cells reduced their firing rates or ceased firing altogether.

In the examples shown in Figure 4, both place fields located midway between the box and the goal were greatly attenuated in the three most shortened journey types, whereas place fields in the vicinity of the box were present in all but the most shortened journey types (box-referenced place fields). In contrast, place fields in the vicinity of the fixed food well were present on all five journey types (track- or room-referenced place fields). Some cells had more than one place field. In these cases, subfields closer to the box shifted more than fields farther from the box. An example is shown in Figure 4; the first and last of the firing profiles along the outbound journey in the bottom panel for DG belong to the same PG cell (in dark gray). These two profiles show different place field shifts on the shortened journeys; i.e., although the location of the first firing profile is strongly dependent on the location of the box, the location of the second firing profile is stable across journey types. The place fields in this figure were recorded in the dark. A similar dependence of place field shift on place field position on the track was observed in the light condition in this study and in the study of Gothard et al. (1996b).

As described previously (Gothard et al., 1996b) a comparable pattern of place field shifts was observed on the inbound journeys (data not shown), which began when the rat moved away from the fixed food well (goal) and ended at the food well in the box. On the inbound journeys, in which the origin of the journey was the
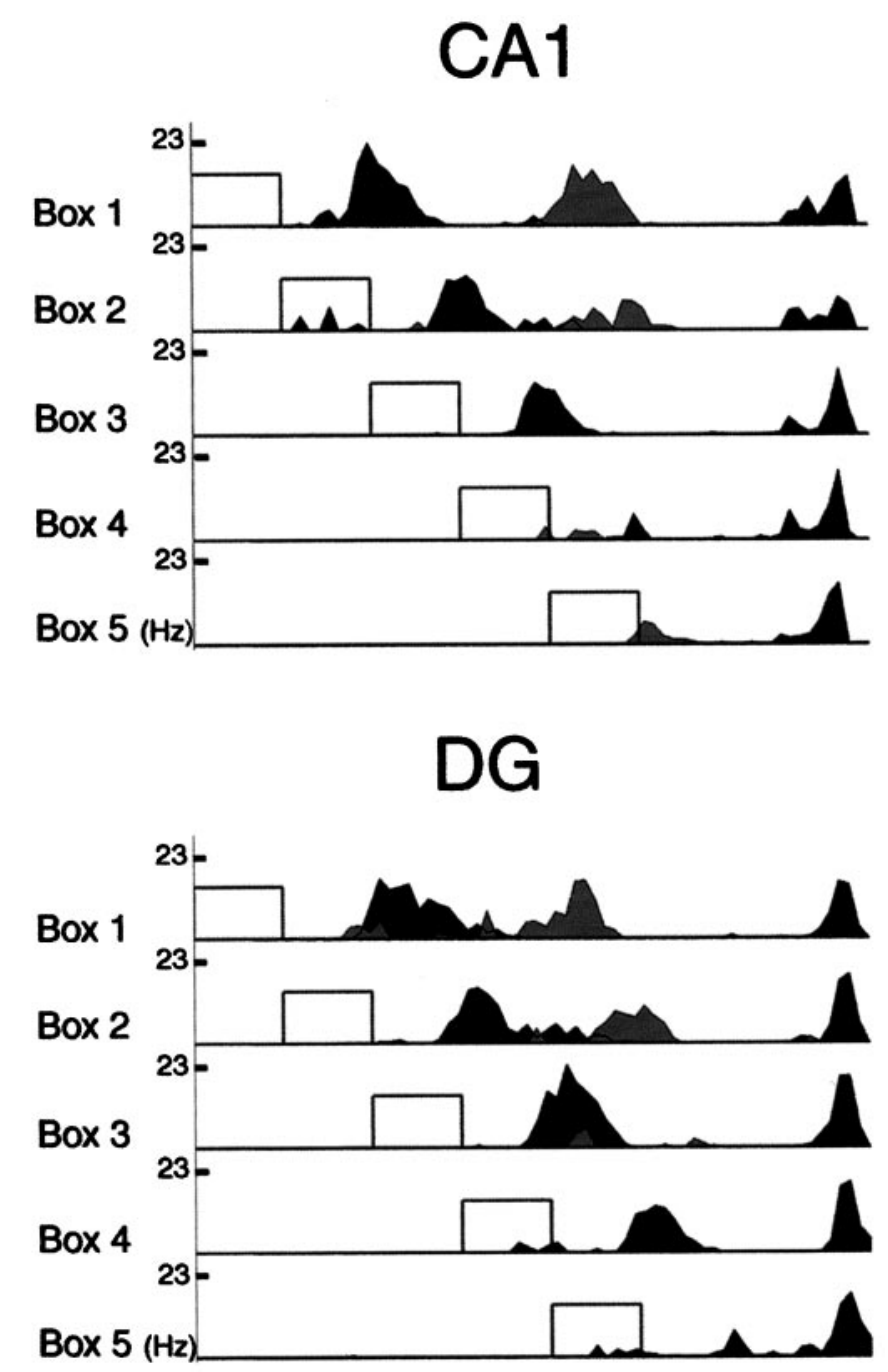

Figure 4. Spatial firing profiles of DG cells and CA1 pyramidal cells on outbound journeys in the dark. Top, Three firing profiles of CA1 pyramidal cells shown for all five types of outbound journeys in the dark. Profiles were calculated by averaging firing rate along the track across all trials of a journey type and normalizing for occupancy. Note that the firing profiles near the box remain aligned with the box across most journey types. In contrast, the firing profiles at the end of the track remain stationary across journey types. The firing profile in the middle of the box 1 out journey shifted less than the firing profile in the vicinity of the box and disappeared in the three shortest journeys. Bottom, Three firing profiles of DG cells shown for all five types of outbound journeys in the dark. Similar to the firing profiles seen in CA1, DG cell profiles in the vicinity of the box shifted with the box, whereas the profiles at the end of the track remained fixed.

fixed food well and the destination was the shifting box, the transition zone was located closer to the end of the journey, i.e., immediately before the entrance to the box. Consistent with the detailed description of directionality by Gothard et al. (1996b) and Jung and McNaughton (1993), most of the CA1 and PG cells had directional place fields. Bidirectional fields occurred no more than expected by chance, given the (low) probability of multiple, independent fields within the same environment.

The extent to which a place field represents location in the reference frame of the box or of the track can be expressed as the slope of the lines in Figure 5, where a slope of 1 corresponds to a place field that maintains constant relationship with the box, 

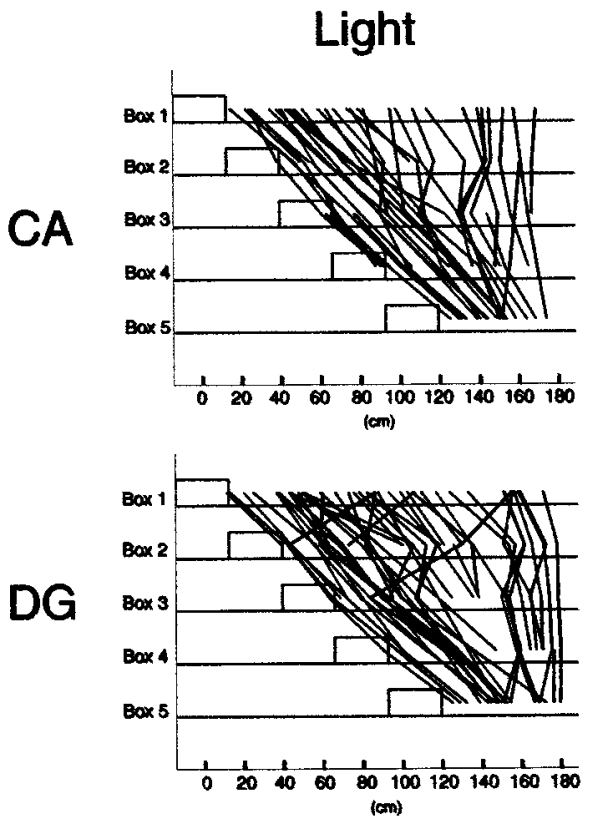

Table 1. Means and SDs of slopes calculated for CA1 and DG place fields on the first and second halves of the track

\begin{tabular}{llllll} 
& \multicolumn{2}{l}{ CA1 fields } & & DG fields & \\
\cline { 2 - 3 } \cline { 5 - 6 } \cline { 5 - 6 } & Light & Dark & & Light & Dark \\
\hline First half & $0.77 \pm 0.27$ & $0.82 \pm 0.19$ & & $0.81 \pm 0.22$ & $0.87 \pm 0.19$ \\
Second half & $0.22 \pm 0.22$ & $0.18 \pm 0.23$ & & $0.21 \pm 0.26$ & $0.23 \pm 0.24$
\end{tabular}

The slope was calculated by connecting the centers of mass of the spikes discharged on at least three outbound journeys. A slope of 1 corresponds to a place field that maintains a constant relationship with the box, and a slope of 0 corresponds to a place field that is fixed in the reference frame of the track and the surrounding fixed cues. Note that on the first half of the journey the slopes have higher values, indicating that the place fields remain aligned with the box, whereas on the second half they tended to align with the fixed reference frame.

and a slope of 0 corresponds to a place field that is fixed in the reference frame of the track.

The slope was calculated for those cells that had a place field in at least three of the five journeys. The mean and SD of the slopes for the first and second halves of the outbound journeys calculated separately for the light and dark conditions and for CA1 and DG are presented in Table 1. The slope values indicate that on the first half of the journey, fields tended strongly to remain aligned with the box, whereas on the second half, they tended to align with the fixed reference frame.

\section{Population vector analysis}

The similarity of the population activity across journey types was quantified by point-by-point correlation of the population vectors computed for each spatial location (see Materials and Methods). In both illumination conditions, cells fired in the same spatial order between the box and the goal; however, on the shortened journeys, some place fields decreased in size and firing rates, or were entirely absent, skipping over some cells in the firing sequence. The least variability was observed near the fixed food well, whereas the maximum variability was in the intermediate zone. In both CA1 and DG, for both illumination conditions, ensemble activity near the beginnings of the shortened journeys was highly correlated with the ensemble activity near the beginnings of the full-length journey, whereas the activity patterns near
Figure 5. Place field alignments on outbound journeys. Centers of mass of firing rate distributions of each principal cell were calculated separately for the five outbound journey types. The centers of mass across journey types for each cell are connected by lines. Consistent with the individual examples in Figure 4, cellular activity remained aligned with the box for a large extent of the journey. Some cells active in the midportion of the journey were silent on the shorter journeys. This shift in activity occurred in both CA1 and DG in both light and dark conditions. The top left plot was constructed from 144 CA1 pyramidal cells from the light condition, and the top right plot was constructed from 157 CA1 pyramidal cells from the dark condition. The bottom plots contain the corresponding plots for the DG light (125 cells) and dark (79 cells) conditions. Note that this measure does not take into account situations in which a single cell had more than one place field on the track. As shown previously (Gothard et al.; 1996), in such cases, place fields tended to behave independently, in a manner that depended on their location on the full track. Thus, the population vector correlations shown in Figure 6 provide a more robust representation of the coordinate frame shift dynamics. the ends of the shortened journeys were correlated more with the patterns near the end of the full-length journey. This is indicated by the reference lines in Figure 6.

\section{Comparison of place field shifts in the light and dark conditions}

Although the rats ran slower in the dark than in the light, no differences in place field size (based on the box 1 trials) and firing rate were observed between these two conditions (two-tailed $t$ test, $p>0.7)$. The box was not visible in the dark; nevertheless, during the outbound journeys, place fields remained aligned with the box for considerable distances (up to $1 \mathrm{~m}$ from the box). The fact that fields remained aligned with the box well beyond the box boundaries under both light and dark conditions is illustrated in Figures 4 and 5. Place fields in the immediate vicinity of the fixed food well remained aligned with the track.

\section{Trial-by-trial analysis of the transition points in the light and dark conditions}

Given that place fields of CA1 and DG cells showed a similar pattern of shift along the track, the spike trains from CA1 and DG were pooled to calculate a coherency measure, i.e., the extent to which the spatial representation contained in the firing of all hippocampal cells is tied to one or the other reference frame. On the basis of the coherence, the transition points between box and track alignment were computed for each trial (see Materials and Methods). The distributions of transition points in the dark and light conditions are shown in Figure 7.

In the dark condition as in the light condition, for every $27 \mathrm{~cm}$ shift of the box, the transition points shifted $\sim 15 \mathrm{~cm}$ farther on the track. The relationship between the translation of the start box and the shift of the transition point appeared to be linear (Fig. 7), with the exception of the box 5 out journeys in the dark. On the shortest, box 5 out, journey in the dark, the distribution of transition points overlapped with the distribution of transition points on the box 4 out journeys. On the contrary, in the light, the transition points shifted proportionally with each box location; i.e., the transition occurred at approximately equal increments for each increment in 

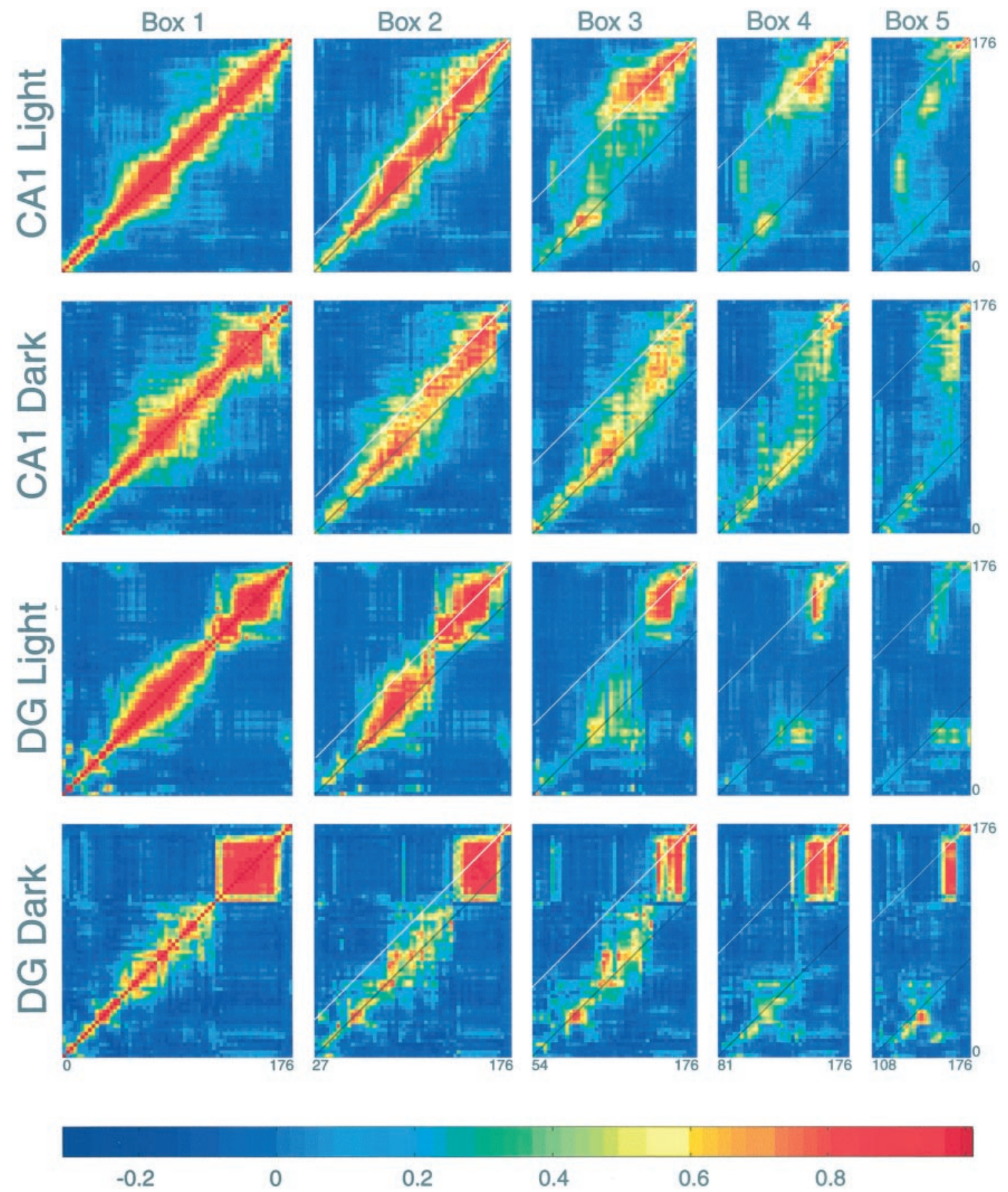

Figure 6. Population vector correlations between full-length and shortened outbound journeys for CA1 and DG cells. For each outbound journey type, a correlation coefficient was calculated for the population vector at each location on the shortened journey $(x$-axis) with each population vector on the full track ( $y$-axis), creating five correlation matrices for each experimental condition and cell class. The four rows of correlation matrices are constructed from the activities of 144, 157, 125, and 79 cells, respectively. The Box 1 matrix is simply the correlation of each population vector on the full track with every other vector on the full track. The broad region of higher correlation along the diagonal reflects the fact that neighboring spatial locations tend to have correlated firing patterns. The black diagonal line indicates the box reference frame, and the white line corresponds to the laboratory reference frame. Note that in all cases, the vector correlations undergo a transition from the box to the laboratory reference frames. Only at the end of the journey was the population activity consistent with absolute position. This indicates that population activity was aligned with the box well after the rat had left the box and that in both regions, this maintained alignment occurred even under dark conditions. The color bar provides the correlation scale. The distance scale is in centimeters.

box position (Fig. 7, solid lines). Most importantly, with the exception of the shortest journeys, the transition points in the dark occurred significantly farther along the journey than in the light $(p<0.01)$, indicating that whatever factor or process maintained box-referenced firing in the light persisted longer in the dark.

\section{DISCUSSION}

In both light and dark conditions, place fields of both CA1 and PG cells exhibited persistent activity in the reference frame of the moveable box, i.e., the point of departure on each journey. Consistent with the behavior of CA1 ensembles reported by Gothard et 
A

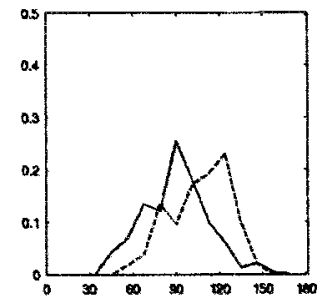

D

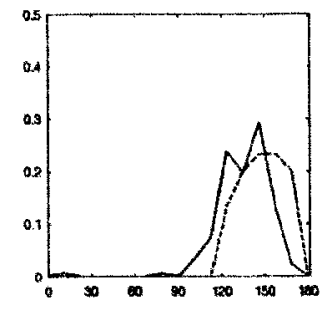

B

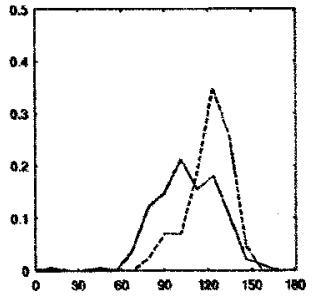

$\mathbf{E}$

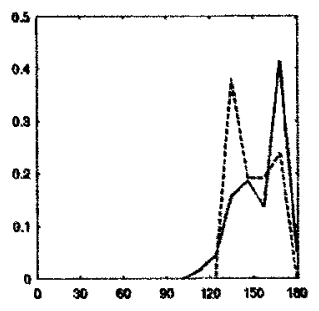

C

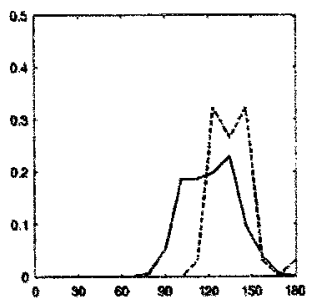

F

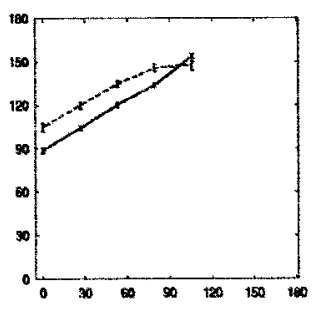

Figure 7. Distribution of transition points in the dark and light. The transition from the box reference frame to the laboratory reference frame was determined using a procedure (Redish et al., 2000; see Materials and Methods) in which the tightness of the place field distribution in the two reference frames was computed at each moment in time. The transition was taken as the point where the difference between the two measures crossed zero. $A-E$, Histograms of transition points in the light (solid lines) and dark (dotted lines) for box 1 out-box 5 out journeys, respectively. The $x$-axis represents the track (in centimeters), and the $y$-axis represents normalized counts. $F$ shows the location of the mean transition points on the track. The $x$-axis corresponds to the origin of a journey on the track, and the $y$-axis represents the location of the mean transition points $( \pm$ SEM) in the light (solid lines) and dark (dotted lines) conditions for each of the five journey types. Box location and transition point are related linearly, with the exception of the transition points for the box 5 out journeys in the dark, for which the transition occurred earlier than expected on the basis of the first four journey types. al. (1996b), place fields in both CA1 and DG showed a dynamic shift from box-aligned ensemble activity to activity aligned to the reference frame of the fixed landmarks in the environment. The transition between reference frames was nonlinear in the sense that the ensemble remained aligned with the box for a substantial portion of the track, despite the fact that the box was well behind the rat (more than two body lengths including the tail) or invisible to the rat (in the dark) by the time the transition to the room frame occurred. The shift appeared in the population as a whole, not independently in different sets of cells. As suggested by Gothard et al. (1996b) the maintained alignment of place fields to a boxrelated reference frame on the outbound journeys might be the result of an internal dynamic process such as path integration. The present results reinforce the conclusion that the correlation of hippocampal ensemble activity with location can be accurately updated by path integration (McNaughton et al., 1989; Muller et al., 1991; Gothard et al., 1996a; Recce and Harris, 1996; Touretzky and Redish, 1996; Samsonovich and McNaughton, 1997; Whishaw, 1998; Mittelstaedt, 2000).

The principal finding of this study is that the box-referenced activity persisted significantly longer in the dark. These results support the hypothesis that the persistence of box-related activity is not attributable to visual signals from the box; rather, it is maintained or updated by self-motion signals. The relative preponderance of self-motion signals over external sensory signals in the dark, without loss of accuracy of the population code for place, strongly suggests that place fields are controlled dynamically by whatever signals are available and reliable in any situation. Because the box was moved relative to the rail, there was no possibility that the rat could have used local sensory cues on the rail to maintain place field alignment in the box reference frame. An olfactory gradient emanating from the box or some form of echolocation are also implausible, because the transition on the return journeys did not occur until the animal was at or near the threshold of the box (i.e., much closer to the box than on the outbound journey). Although visual and olfactory cues may not account for the persistence of activity in the reference frame of the box, the present data leave open the possibility that this effect is not a consequence of path integration per se. The persistence of ensemble activity aligned with the box might reflect the replay of a sequence of states that was learned during the animals' initial training in the light with the box in a fixed location. Thus, the context of leaving the box may be represented in the hippocampus by some time-dependent sequence of states that is not tightly bound to any physical location. Redish et al. (2000) have obtained similar results (in the light) under conditions in which the rat never received initial training with the box at a fixed location, making an explanation based on previously learned sequences of external inputs unlikely; however, the existence of some strictly time-dependent sequence cannot be entirely ruled out with the present data.

The fact that the transition in the dark nevertheless occurred before the rat reached the fixed goal at the track end probably reflects a combination of experience with the task and the presence of local cues on the track, which were fixed in the laboratory reference frame. It is plausible that during the 1000-1500 training trials that preceded most recording sessions, the rats learned the expected distances between all the possible box locations and the goal. This possibility is supported by the rats' behavior during a probe trial in the dark, when a baited food cup, identical to the fixed food cup at the goal, was moved halfway between the box and the goal. The rat ran over the food cup, ignoring the olfactory and tactile cues associated with it, and stopped at the end of the track to eat the bait from the fixed food cup.

Finally, the similarity between the place field shifts in the CA1 and dentate gyrus ensembles rules out any simple, open-loop, sequential processing hypothesis in which the path integration operation is performed in $\mathrm{CA} 3$, and the result is transferred unidirectionally to $\mathrm{CA} 1$ and projected out of the hippocampus without recirculating via the entorhinal cortex or other intrinsic connections. Indeed, the present result raises the possibility that the dentate gyrus itself may be capable of updating the hippocampal ensemble on the basis of self-motion information. Verification of this hypothesis would require comparison of the behavior of PG cells and projection cells from entorhinal layer II.

Other possibilities also remain. The simplest explanation for these effects is that the hippocampus does not take part in the path integration computation at all but merely receives path integration information as a component of its input (Touretzky and Redish, 1996; Arbib, 1997; Cooper and Mizumori, 1999; Sharp, 1999). The nature and site of implementation of the path integration computations remain to be elucidated. At the behavioral level, there are mixed results in the literature concerning the necessity of an intact hippocampus for path integration (Whishaw 
and Jarrard, 1996; Alyan and McNaughton, 1999; Maaswinkel et al., 1999); however, it is known that the output of CA1 feeds back to the entorhinal cortex and also that CA3 sends some recurrent excitatory projections directly back to the dentate gyrus (Penttonen et al., 1997). It may well be that these longer-range recurrent connections serve to maintain a consistent representation throughout all principal hippocampal subfields.

\section{REFERENCES}

Alyan S, McNaughton BL (1999) Hippocampectomized rats are capable of homing by path integration. Behav Neurosci 113:19-31.

Arbib MA (1997) From visual affordances in monkey parietal cortex to hippocampo-parietal interactions underlying rat navigation. Philos Trans R Soc Lond [Biol] 352:1429-1436.

Bragin A, Jando G, Nadasdy Z, van Landeghem M, Buzsaki G (1995) Dentate EEG spikes and associated interneuronal population bursts in the hippocampal hilar region of the rat. J Neurophysiol 73:1691-1705.

Bures J, Fenton AA, Kaminsky Y, Zinyuk L (1997) Place cells and place navigation. Proc Natl Acad Sci USA 94:343-350.

Buzsaki G (1986) Hippocampal sharp waves: their origin and significance. Brain Res 398:242-252.

Buzsaki G, Leung LW, Vanderwolf CH (1983) Cellular bases of hippocampal EEG in the behaving rat. Brain Res 287:139-171.

Cooper BG, Mizumori SJ (1999) Retrosplenial cortex inactivation selectively impairs navigation in darkness. NeuroReport 10:625-630.

Etienne AS, Maurer R, Seguinot V (1996) Path integration in mammals and its interaction with visual landmarks. J Exp Biol 199:201-209.

Freund TF, Buzsaki G (1996) Interneurons of the hippocampus. Hippocampus 6:347-470.

Golob EJ, Taube JS (1999) Head direction cells in rats with hippocampal or overlying neocortical lesions: evidence for impaired angular path integration. J Neurosci 19:7198-7211.

Gothard KM, Skaggs WE, Moore KM, McNaughton BL (1996a) Binding of hippocampal CA1 neural activity to multiple reference frames in a landmark-based navigation task. J Neurosci 16:823-835.

Gothard KM, Skaggs WE, McNaughton BL (1996b) Dynamics of mismatch correction in the hippocampal ensemble code for space: interaction between path integration and environmental cues. J Neurosci 16:8027-8040.

Hill AJ, Best PJ (1981) Effects of deafness and blindness on the spatial correlates of hippocampal unit activity in the rat. Exp Neurol 74:204-217.

Jung MW, McNaughton BL (1993) Spatial selectivity of unit activity in the hippocampal granular layer. Hippocampus 3:165-182.

Knierim JJ, Kudrimoti HS, McNaughton BL (1995) Place cells, head direction cells, and learning of landmark stability. J Neurosci 15:1648-1659.

Knierim JJ, Kudrimoti HS, McNaughton BL (1998) Interactions between idiothetic cues and external landmarks in the control of place cells and head direction cells. J Neurophysiol 80:425-446.

Maaswinkel H, Jarrard LE, Whishaw IQ (1999) Hippocampectomized rats are impaired in homing by path integration. Hippocampus 9:553-561.

Markus EJ, Barnes CA, McNaughton BL, Gladden VL, Skaggs WE
(1994) Spatial information content and reliability of hippocampal CA1 neurons: effects of visual input. Hippocampus 4:410-421.

McNaughton BL, Barnes CA, O'Keefe J (1983) The contributions of position, direction, and velocity to single unit activity in the hippocampus of freely-moving rats. Exp Brain Res 53:41-49.

McNaughton BL, Leonard B, Chen L (1989) Cortical-hippocampal interactions and cognitive mapping: a hypothesis based on re-integration of the parietal and inferotemporal pathways for visual processing. Psychobiology 17: 230-235.

Muller RU, Kubie JL (1987) The effects of changes in the environment on the spatial firing of hippocampal complex-spike cells. J Neurosci 7:1951-1968

Muller RU, Kubie JL, Saypoff R (1991) The hippocampus as a cognitive graph (abridged version). Hippocampus 3:243-246.

Nitz DA, McNaughton BL (1999) Hippocampal EEG and unit activity responses to modulation of serotonergic median raphe neurons in the freely behaving rat. Learn Mem 6:153-167.

O'Keefe J (1976) Place units in the hippocampus of the freely moving rat. Exp Neurol 51:87-109.

O'Keefe J, Burgess N (1996) Geometric determinants of the place fields of hippocampal neurones. Nature 381:425-428.

O'Keefe J, Conway DH (1978) Hippocampal place units in the freely moving rat: why they fire where they fire. Exp Brain Res 31:573-590.

Penttonen M, Kamondi A, Sik A, Acsady L, Buzsaki G (1997) Feedforward and feed-back activation of the dentate gyrus in vivo during dentate spikes and sharp wave bursts. Hippocampus 7:437-450.

Quirk GJ, Muller RU, Kubie JL (1990) The firing properties of hippocampal place cells in the dark depends on the rat's recent experience. J Neurosci 10:2008-2017.

Ranck Jr JB (1973) Studies on single neurons in dorsal hippocampal formation and septum in unrestrained rats. Exp Neurol 41:461-531.

Recce M, Harris KD (1996) Memory for places: a navigational model in support of Marr's theory of hippocampal function. Hippocampus 6:735-748.

Recce ML, O'Keefe J (1989) The tetrode: an improved technique for multi-unit extracellular recording. Soc Neurosci Abstr 15:1250.

Redish AD (1999) Beyond the cognitive map: from place cells to episodic memory. Cambridge, MA: MIT.

Redish AD, Rosenzweig ES, Bohanick JD, McNaughton BL, Barnes CA (2000) Dynamics of hippocampal ensemble activity realignment: time versus space. J Neurosci 20:9298-9309.

Samsonovich A, McNaughton BL (1997) Path integration and cognitive mapping in a continuous attractor neural network model. J Neurosci 17:5900-5920.

Sharp PE (1999) Comparison of the timing of hippocampal and subicular spatial signals: implications for path integration. Hippocampus 9:158-172.

Stumpf C (1965) Drug action on the electrical activity of the hippocampus. Int Rev Neurobiol 8:77-138.

Touretzky DS, Redish AD (1996) Theory of rodent navigation based on interacting representations of space. Hippocampus 6:247-270.

Whishaw IQ (1998) Place learning in hippocampal rats and the path integration hypothesis. Neurosci Biobehav Rev 22:209-220.

Whishaw IQ, Jarrard LE (1996) Evidence for extrahippocampal involvement in place learning and hippocampal involvement in path integration. Hippocampus 6:513-524.

Wilson MA, McNaughton BL (1993) Dynamics of the hippocampal ensemble code for space. Science 261:1055-1058. 